Mol Neurobiol. 2011 December ; 44(3): 420-425. doi:10.1007/s12035-011-8208-y.

\title{
Phosphoinositides and Photoreceptors
}

\author{
Susan E. Brockerhoff \\ Department of Biochemistry, University of Washington, Seattle, WA 98195, USA
}

\begin{abstract}
The importance of phosphoinositides (phosphorylated phosphatidyl inositol derivatives, PIs) for normal cellular function cannot be overstated. Although they represent a small fraction of the total phospholipid within the cell, they are essential regulators of many cellular functions. They direct membrane trafficking by functioning as recruitment factors for vesicular trafficking components, they can modulate ion channel activity through direct binding within cellular membranes, and their hydrolysis generates second messenger signaling molecules. Despite an explosion of information regarding the importance of these lipids in cellular biology, their precise roles in vertebrate retinal photoreceptors has not been established. This review summarizes the literature on potential roles for different phosphoinositides and their regulators in vertebrate rods and cones. A brief description of the importance of PI signaling in other photosensitive cells is also presented. The highly specialized functions of the vertebrate photoreceptor, combined with the established importance of phosphoinositides, promise significant future discoveries in this field.
\end{abstract}

\section{Keywords}

Retina; Photoreceptor; Phosphoinositides; Synaptojanin

\section{Photoreceptors}

Retinal rod and cone photoreceptors are highly specialized sensory neurons with a polarized structure. They contain an outer segment with membranous discs housing the proteins needed for phototransduction, an inner segment where the cell machinery for protein synthesis and energy production resides, and a unique ribbon-type synapse specialized for graded continuous release of the neurotransmitter glutamate. Key features of normal photoreceptor activity are light-mediated signaling, regulated channel activity, and directed vesicular trafficking. These processes are highly dependent on phosphoinositides. Imbalances lead to severe defects that cause abnormalities in photoreceptor function and formation and consequent blindness. For example, loss of the $\mathrm{PI}(4,5) \mathrm{P}_{2}$ phosphatase synaptojanin 1 (SynJ1) causes major structural abnormalities in zebra fish cone synaptic pedicles contributing to visual impairment and abnormal electroretinograms (ERGs) in these fish [1]. In addition, conditional deletion of the p $85 \alpha$ regulatory subunit of phosphoinositide 3-kinase (PI3K) in mouse cones leads to slow degeneration of these cells [2].

\section{Phosphoinositides in Phototransduction}

Phosphoinositide signaling has been implicated in modulating vertebrate phototransduction. Light increases the synthesis of phosphoinositides within photoreceptors [3, 4], increases phospholipase C (PLC) activity in outer segments [5, 6], enhances uptake of $\left[{ }^{3} \mathrm{H}\right]$ inositol

(C) Springer Science+Business Media, LLC 2011

sbrocker@uw.edu . 
and PI turnover in photoreceptors $[3,7]$ and can lead to the release of inositol triphosphate (IP3) from the retina [8]. Further, immunocytochemical studies analyzing rod outer segments have detected IP3 receptors [9], PLC [10, 11], and Ga11 [11]. One potential role for a PLC signaling cascade is the light-dependent regulation of arrestin translocation from inner to outer segments. Activators of both PKC and PLC initiate arrestin translocation to the outer segments in the absence of light, and antagonists of these enzymes reduce the light-activated movement of arrestin to outer segments [12]. Physiological studies also support the idea that phosphoinositides are involved in modulating phototransduction. Studies examining the activity of phosphodiesterase (PDE) and rod cyclic nucleotide-gated channels from Xenopus photoreceptors indicate that exogenous $\mathrm{PI}(4,5) \mathrm{P}_{2}$ can enhance PDE activation by nucleotides and inhibit channel activity [13]. Together these data suggest important modulatory roles for phosphoinositides in outer segments, however precise molecular mechanisms and cascades still need to be determined.

\section{Phosphoinositide 3-kinase Signaling in Outer Segments}

In addition to modulating phototransduction, phosphoinositide signaling in outer segments is implicated in pathways essential for cell survival. Rajala and colleagues have collected evidence for a rhodopsin and light-dependent cascade leading to activation of PI3K/AKT signaling promoting enhanced rod photoreceptor survival (reviewed in [14, 15]). The current model is that light-dependent but insulin-independent phosphorylation of the insulin receptor (IR) causes an increase in $\mathrm{PI}(3,4,5) \mathrm{P}_{3}$ due to the activation of PI3K [16]. This activity requires photobleaching of rhodopsin but not activation of rod transducin [17] and may be mediated by the growth factor receptor-bound protein 14 (GRB14) since ablation of GRB14 resulted in the loss of light-dependent kinase activity of retinal IR [18]. One possibility is that GRB14 reduces dephosphorylation of the insulin receptor in photoreceptors as proposed from in vitro studies [19]. GRB14 has also been shown to bind to the rod photoreceptor-specific cyclic nucleotide gated channel alpha subunit decreasing its affinity for cyclic guanosine monophosphate [20]. Surprisingly however, no differences in retinal morphology or ERG waveforms were detected in GRB14-/- mice [18]. In addition, conditional cell-type specific ablation of the p $85 \alpha$ regulatory subunit of phosphoinositide 3-kinase caused slow degeneration of cones [2] but not rods [21]. However, mice lacking the insulin receptor specifically in rods showed dramatic losses in photoreceptors when exposed to bright light stress [22]. Together these findings suggest important regulatory functions for a PI3K-mediated pathway in photoreceptor outer segments that may involve redundant pathways and differential regulation in rods versus cones.

\section{Phosphoinositides in Protein Transport and Sorting in the Inner Segment}

The elongated and polarized morphology of vertebrate photoreceptors is created and maintained by precisely regulated vesicular trafficking originating in the inner segment. This process occurs in two directions, apically toward the outer segment and basally toward the synapse. Rhodopsin is the major protein component in outer segments, and phosphoinositides are key regulators of vesicle trafficking required for its transport. Rhodopsin transport initiates at the Golgi. Recent work shows that the PI $(4,5) \mathrm{P}_{2}$ binding protein ASAP1 (Arf GAP with SH3 domain, ankyrin repeat, and PH domain 1) is required for efficient budding of rhodopsin transport carriers at the Golgi [23]. ASAP1 is thought to serve both as an Arf4 GAP and an Arf4 effector. ASAP1 facilitates formation of a protein complex containing Rab11, FIP3, and Arf4. The functional site of this complex is at the trans-Golgi network (TGN), where it regulates the packaging of rhodopsin into post-TGN carriers targeted to the photoreceptor cilia [23]. 
After transport from the Golgi, phosphatidylinositol 3-phosphate (PI3P) becomes key in rhodopsin trafficking. Rhodopsin transport and disc biogenesis depends on the PI3P binding protein smad anchor for receptor activation (SARA) and the PI3P binding capabilities of SARA are essential for these processes [24]. Immature disc membranes contain an abundance of PI3P and the fusion of rhodopsin-laden vesicles with these membranes is mediated by FYVE, the PI3P lipid-binding domain of SARA. SARA binds directly to the Cterminus of rhodopsin and to the SNARE protein syntaxin 3. Depletion of PI3P binding sites within photoreceptors by overexpression of the lipid-binding domain FYVE causes mislocalization of endogenous rhodopsin as well as abnormal vesicle accumulation beneath the most basal discs within the outer segment. One interesting hypothesis based on these studies is that regulation of PI3P levels in forming disc membranes controls disc maturation [24].

Although significantly less is known about basal transport, data from our lab suggest that PIs are critical for this as well. Fish lacking the $\mathrm{PI}(4,5) \mathrm{P}_{2}$ phosphatase SynJ1 develop morphologically normal outer segments [25] with normal ERG a-wave responses in the absence of background illumination [26] suggesting that apical transport does not depend on this protein. However, our recent work shows that vesicles destined for the synapse accumulate in the cone inner segments in SynJ1 mutants and that SynJ1 protein localizes to this region within cone photoreceptors (Fig. 1 and [27]). These data suggest the interesting possibility that phosphoinositides, possibly $\mathrm{PI}(4,5) \mathrm{P}_{2}$, regulate the sorting of synaptic proteins in cone photoreceptor inner segments (Fig. 2).

\section{Phosphoinositides at the Photoreceptor Synapse}

Synapses of vertebrate sensory neurons, such as photoreceptors, contain structures called synaptic ribbons. These elongated electron-dense scaffolds tether a pool of releasable synaptic vesicles at active zones. Although their precise function is still uncertain, they are important for the tonic rapid release required for sensory perception (for review, see [28]). Consistent with what is known about synaptic function and vesicle recycling at conventional neurons, phosphoinositides are key to maintaining normal photoreceptor synapse function and morphology. One possible function for phosphoinositide signaling is in the diurnal regulation of ribbon length. These phenomena are well-documented in many fish species and may be an energy-conserving mechanism that decreases visual sensitivity at night [29, 30]. Studies in carp retina showed that PI metabolism was activated in photoreceptor synapses during dark adaptation and treatment of retinas with IP3 induced a decrease in the number of synaptic ribbons [31].

Studies in zebra fish also emphasize the importance of phosphoinositides, particularly $\mathrm{PI}(4,5) \mathrm{P}_{2}$, at photoreceptor synapses. Zebra fish lacking SynJ1 have many morphological defects at the cone photoreceptor synapse. These include an overall flattened (uninvaginated) morphology, clustered vesicles, reduced vesicle number, as well as elongated and predominantly unanchored synaptic ribbons (Fig. 2 and [1]). Consistently, this mutant has abnormal visual responses and electroretinograms [25, 26]. Further, expression studies using a fluorescently tagged pleckstrin homology domain selective for $\mathrm{PI}(4,5) \mathrm{P}_{2}$ in cone photoreceptors show an abundance of this lipid at the synapse (Fig. 3).

Interestingly, these studies also suggest that phosphoinositides are more important for correct synaptic physiology in cones than in rods. The effect on ribbon structure in carp retinas by IP3 addition was only observed in cones and not rods [31] and rod spherules from SynJ1-defective zebra fish mutants are reported as morphologically normal consistent with the lack of expression of SynJ1 protein in zebra fish rod spherules and striking abundance of this protein in cone pedicles [27]. 


\section{The Role of PIs in Other Photosensitive Cells}

Although this review focuses on PI metabolism in vertebrate retinal photoreceptors, it is important to note that PI signaling is also critical for the function of other vertebrate and invertebrate photosensitive cells. A primary example of this is the Drosophila photoreceptor. In this cell, phototransduction does not depend on cGMP hydrolysis but is instead mediated by the hydrolysis of PI $(4,5) \mathrm{P}_{2}$ by PLC into IP3 and DAG (reviewed in [32-34]). This G protein-coupled PLC signaling cascade leads to the opening of two classes of calcium channels called trp and trp-like. The calcium influx leads to cell depolarization and affects multiple targets in signal amplification, rapid response termination, and light adaptation. This sensory cascade mediates extremely fast responses to visual stimuli. Another example is the vertebrate intrinsically photosensitive ganglion cell (ipRGCs). Current evidence indicates that at least some photosensitive ganglion cell subtypes have phototransduction systems that are similar to the Drosophila photoreceptor (for review, see [35]). In ipRGCs, the photopigment melanopsin, which resembles invertebrate rhodopsins, couples to a Gqtype G protein and signals to a PLC to open trp channels. These similarities in the signaling cascades suggest that the Drosophila and mammalian ipRGC phototransduction cascades are evolutionarily related.

\section{Summary}

Many studies have demonstrated a key role for phosphoinositide metabolism in many aspects of vertebrate rod and cone photoreceptor function. Phosphoinositides modulate photoresponses and regulate apical and possibly basal vesicular transport, PI3K-mediated signaling cascades influence survival, and imbalances in phosphoinositide homeostasis dramatically alter synapse function and morphology. Future studies in this important research area will continue to define the mechanism of phosphoinositide function and regulation. One particularly interesting aspect of these studies will be an analysis of differences between rods and cones since current data suggest that phosphoinositide metabolism may be distinctly different between these cell types.

\section{Acknowledgments}

The author's research is funded by NIH grants EY015165 and EY018814. Thanks to Ashley George and Dr. Sara Hayden for the comments on the manuscript.

\section{References}

1. Van Epps HA, Hayashi M, Lucast L, Stearns GW, Hurley JB, De Camilli P, Brockerhoff SE. The zebrafish nrc mutant reveals a role for the polyphosphoinositide phosphatase synaptojanin 1 in cone photoreceptor ribbon anchoring. J Neurosci. 2004; 24:8641-8650. [PubMed: 15470129]

2. Ivanovic I, Anderson RE, Le YZ, Fliesler SJ, Sherry DM, Rajala RV. Deletion of the p85alpha regulatory subunit of phosphoinositide 3-kinase in cone photoreceptor cells results in cone photoreceptor degeneration. Invest Ophthalmol Vis Sci. 2011; 52:3775-3783. [PubMed: 21398281]

3. Schmidt SY. Light- and cytidine-dependent phosphatidylinositol synthesis in photoreceptor cells of the rat. J Cell Biol. 1983; 97:832-837. [PubMed: 6885920]

4. Ghalayini AJ, Anderson RE. Light adaptation of bovine retinas in situ stimulates phosphatidylinositol synthesis in rod outer segments in vitro. Curr Eye Res. 1995; 14:1025-1029. [PubMed: 8585931]

5. Ghalayini A, Anderson RE. Phosphatidylinositol 4,5-bisphosphate: light-mediated breakdown in the vertebrate retina. Biochem Biophys Res Commun. 1984; 124:503-506. [PubMed: 6093803]

6. Ghalayini AJ, Weber NR, Rundle DR, Koutz CA, Lambert D, Guo XX, Anderson RE. Phospholipase Cgamma1 in bovine rod outer segments: immunolocalization and light-dependent binding to membranes. J Neurochem. 1998; 70:171-178. [PubMed: 9422360] 
7. Schmidt SY. Light enhances the turnover of phosphatidylinositol in rat retinas. J Neurochem. 1983; 40:1630-1638. [PubMed: 6854323]

8. Jung HH, Reme CE, Pfeilschifter J. Light evoked inositol trisphosphate release in the rat retina in vitro. Curr Eye Res. 1993; 12:727-732. [PubMed: 8222733]

9. Day NS, Koutz CA, Anderson RE. Inositol-1,4,5-trisphosphate receptors in the vertebrate retina. Curr Eye Res. 1993; 12:981-992. [PubMed: 8306716]

10. Ghalayini AJ, Tarver AP, Mackin WM, Koutz CA, Anderson RE. Identification and immunolocalization of phospholipase C in bovine rod outer segments. J Neurochem. 1991; 57:1405-1412. [PubMed: 1895111]

11. Peng YW, Rhee SG, Yu WP, Ho YK, Schoen T, Chader GJ, Yau KW. Identification of components of a phosphoinositide signaling pathway in retinal rod outer segments. Proc Natl Acad Sci U S A. 1997; 94:1995-2000. [PubMed: 9050893]

12. Orisme W, Li J, Goldmann T, Bolch S, Wolfrum U, Smith WC. Light-dependent translocation of arrestin in rod photoreceptors is signaled through a phospholipase $\mathrm{C}$ cascade and requires ATP. Cell Signal. 2010; 22:447-456. [PubMed: 19887106]

13. Womack KB, Gordon SE, He F, Wensel TG, Lu CC, Hilgemann DW. Do phosphatidylinositides modulate vertebrate phototransduction? J Neurosci. 2000; 20:2792-2799. [PubMed: 10751430]

14. Rajala RV. Phosphoinositide 3-kinase signaling in the vertebrate retina. J Lipid Res. 2010; 51:422. [PubMed: 19638643]

15. Rajala RV, Anderson RE. Rhodopsin-regulated insulin receptor signaling pathway in rod photoreceptor neurons. Mol Neurobiol. 2010; 42:39-47. [PubMed: 20407846]

16. Rajala RV, McClellan ME, Ash JD, Anderson RE. In vivo regulation of phosphoinositide 3-kinase in retina through light-induced tyrosine phosphorylation of the insulin receptor beta-subunit. J Biol Chem. 2002; 277:43319-43326. [PubMed: 12213821]

17. Rajala A, Anderson RE, Ma JX, Lem J, Al-Ubaidi MR, Rajala RV. G-protein-coupled receptor rhodopsin regulates the phosphorylation of retinal insulin receptor. J Biol Chem. 2007; 282:98659873. [PubMed: 17272282]

18. Rajala A, Daly RJ, Tanito M, Allen DT, Holt LJ, Lobanova ES, Arshavsky VY, Rajala RV. Growth factor receptor-bound protein 14 undergoes light-dependent intracellular translocation in rod photoreceptors: functional role in retinal insulin receptor activation. Biochemistry. 2009; 48:5563-5572. [PubMed: 19438210]

19. Bereziat V, Kasus-Jacobi A, Perdereau D, Cariou B, Girard J, Burnol AF. Inhibition of insulin receptor catalytic activity by the molecular adapter Grb14. J Biol Chem. 2002; 277:4845-4852. [PubMed: 11726652]

20. Gupta VK, Rajala A, Daly RJ, Rajala RV. Growth factor receptor-bound protein 14: a new modulator of photoreceptor-specific cyclic-nucleotide-gated channel. EMBO Rep. 2010; 11:861867. [PubMed: 20890309]

21. Ivanovic I, Allen DT, Dighe R, Le YZ, Anderson RE, Rajala RV. Phosphoinositide 3-kinase signaling in retinal rod photoreceptors. Invest Ophthalmol Vis Sci. 2011; 52:6355-6362. [PubMed: 21730346]

22. Rajala A, Tanito M, Le YZ, Kahn CR, Rajala RV. Loss of neuroprotective survival signal in mice lacking insulin receptor gene in rod photoreceptor cells. J Biol Chem. 2008; 283:19781-19792. [PubMed: 18480052]

23. Mazelova J, Astuto-Gribble L, Inoue H, Tam BM, Schonteich E, Prekeris R, Moritz OL, Randazzo PA, Deretic D. Ciliary targeting motif VxPx directs assembly of a trafficking module through Arf4. EMBO J. 2009; 28:183-192. [PubMed: 19153612]

24. Chuang JZ, Zhao Y, Sung CH. SARA-regulated vesicular targeting underlies formation of the light-sensing organelle in mammalian rods. Cell. 2007; 130:535-547. [PubMed: 17693260]

25. Allwardt BA, Lall AB, Brockerhoff SE, Dowling JE. Synapse formation is arrested in retinal photoreceptors of the zebrafish $n r c$ mutant. J Neurosci. 2001; 21:2330-2342. [PubMed: 11264308]

26. Van Epps HA, Yim CM, Hurley JB, Brockerhoff SE. Investigations of photoreceptor synaptic transmission and light adaptation in the zebrafish visual mutant $n r c$. Invest Ophthalmol Vis Sci. 2001; 42:868-874. [PubMed: 11222552] 
27. Holzhausen LC, Lewis AA, Cheong KK, Brockerhoff SE. Differential role for synaptojanin 1 in rod and cone photoreceptors. J Comp Neurol. 2009; 517:633-644. [PubMed: 19827152]

28. Matthews G, Fuchs P. The diverse roles of ribbon synapses in sensory neurotransmission. Nat Rev Neurosci. 2010; 11:812-822. [PubMed: 21045860]

29. Emran F, Dowling JE. Larval zebrafish turn off their photoreceptors at night. Commun Integr Biol. 2010; 3:430-432. [PubMed: 21057632]

30. Emran F, Rihel J, Adolph AR, Dowling JE. Zebrafish larvae lose vision at night. Proc Natl Acad Sci U S A. 2010; 107:6034-6039. [PubMed: 20224035]

31. Schmitz F, Drenckhahn D. Li(+)-induced structural changes of synaptic ribbons are related to the phosphoinositide metabolism in photoreceptor synapses. Brain Res. 1993; 604:142-148. [PubMed: 8384508]

32. Hardie RC, Raghu P. Visual transduction in Drosophila. Nature. 2001; 413:186-193. [PubMed: 11557987]

33. Wang T, Montell C. Phototransduction and retinal degeneration in Drosophila. Pflugers Arch. 2007; 454:821-847. [PubMed: 17487503]

34. Borst A. Drosophila's view on insect vision. Curr Biol. 2009; 19:R36-R47. [PubMed: 19138592]

35. Schmidt TM, Chen SK, Hattar S. Intrinsically photosensitive retinal ganglion cells: many subtypes, diverse functions. Trends Neurosci. 2011 in press. 

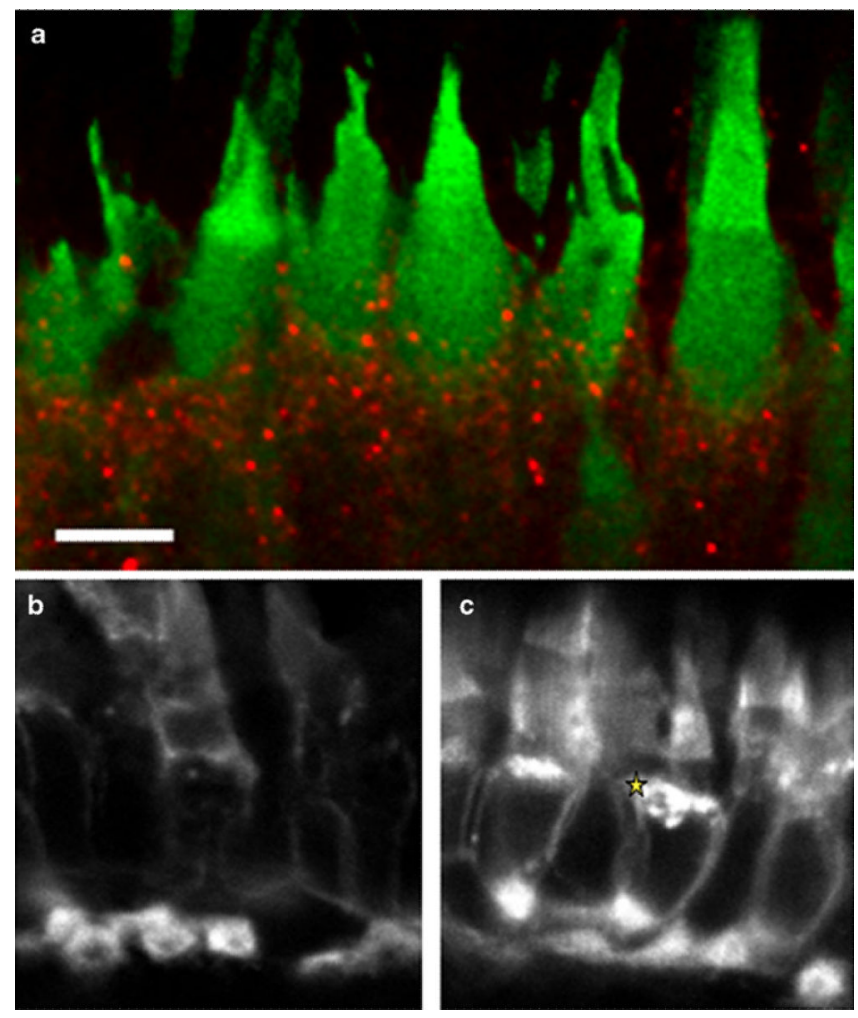

Fig. 1.

Synaptojanin 1 is present in zebra fish cone photoreceptor inner segments and its absence leads to an accumulation of vesicles containing synaptic markers. a Immunocytochemistry showing SynJ1 protein (red) directly beneath outer segments (pseudocolored green) in adult zebra fish cone photoreceptors. b Wild-type larval zebra fish cones expressing synaptopHluorin (VAMP2-tagged pH-sensitive form of GFP), which localizes primarily to the synapse. c Synaptojanin-deficient mutant larval zebra fish cones expressing synaptopHluorin. Note accumulation of vesicles within the inner segment (asterisk). Scale bar $5 \mu \mathrm{m}$. Figure adapted from [27] 

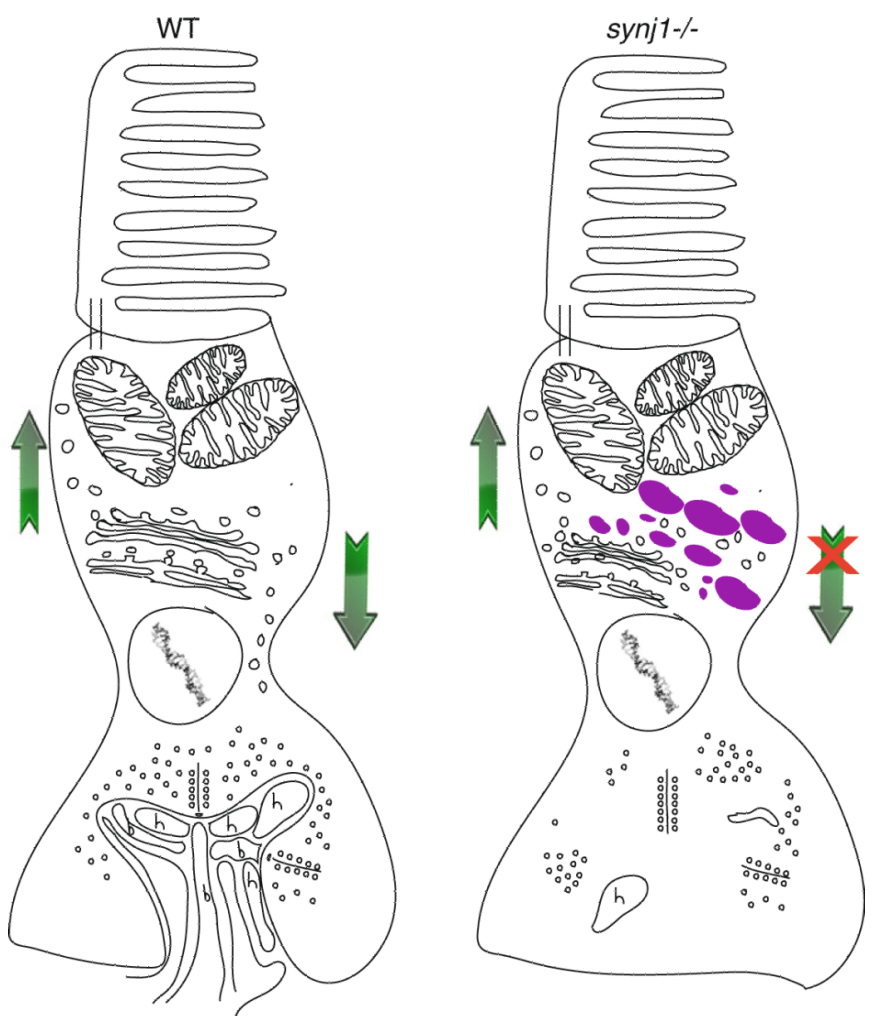

Fig. 2.

Cone photoreceptor phenotypes associated with the loss of the $\mathrm{PI}(4,5) \mathrm{P}_{2}$ phosphatase synaptojanin 1. Zebra fish mutants lacking synaptojanin 1 (synJ1-l-) have major morphological defects at the synapse, however outer segment morphology is normal. In addition, vesicles (purple) containing synaptic proteins accumulate abnormally in mutant inner segments (see text for details). These data suggest a specific defect in transport toward the synapse in mutant cones. Green arrow indicates normal transport. Green arrow with red $X$ indicates disrupted transport. $h$ designates horizontal cell. $b$ designates bipolar cell 


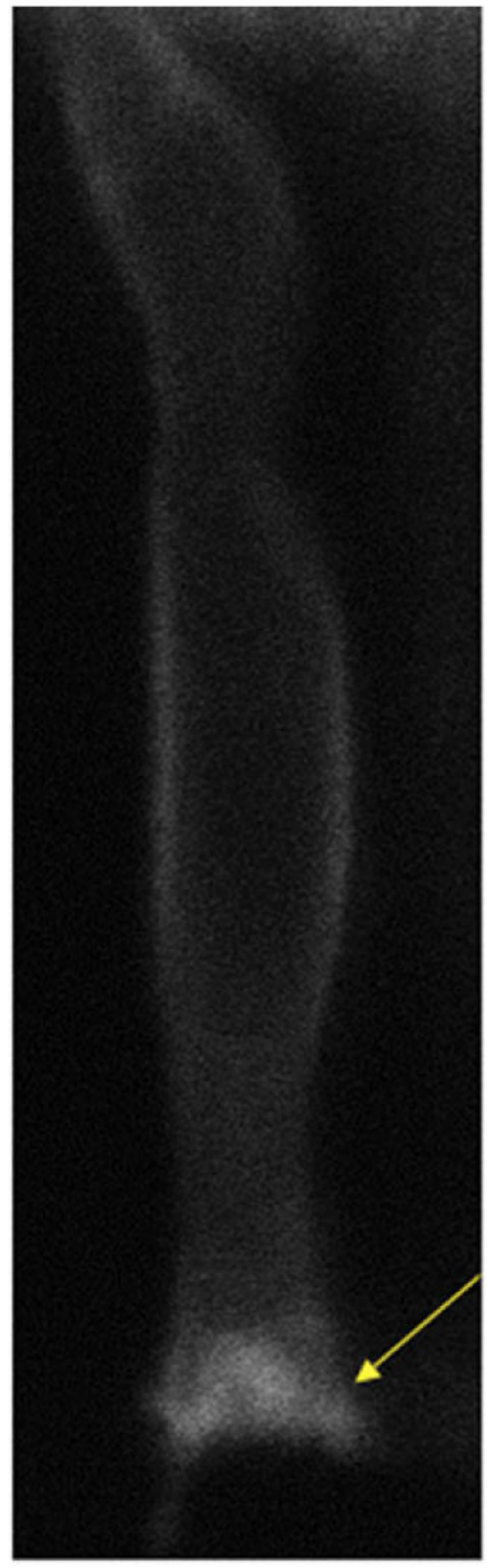

Fig. 3.

Wild type larval zebra fish cones have an abundance of $\mathrm{PI}(4,5) \mathrm{P}_{2}$ at the synapse. A wildtype larval zebra fish cone expressing the $\mathrm{PI}(4,5) \mathrm{P}_{2}$ sensitive pleckstrin homology (PLCdelta) domain attached to CFP shows an abundance of fluorescence at the synapse (arrow, unpublished results) 